\title{
openheart Prognosis and risk stratification in patients with decompensated heart failure receiving inotropic therapy
}

Clara Gomes, ${ }^{1}$ Caíque Bueno Terhoch, ${ }^{2}$ Silvia Moreira Ayub-Ferreira, ${ }^{2}$ Germano Emilio Conceição-Souza, ${ }^{2}$ Vera Maria Cury Salemi, ${ }^{2}$ Paulo Roberto Chizzola, ${ }^{2}$ Mucio Tavares Oliveira $\mathrm{Jr}^{2}$ Silvia Helena Gelas Lage, ${ }^{2}$ Fernando Frioes, ${ }^{1}$ Edimar Alcides Bocchi, ${ }^{2}$ Victor Sarli Issa ${ }^{1,2}$

To cite: Gomes C, Terhoch CB, Ayub-Ferreira SM, et al. Prognosis and risk stratification in patients with decompensated heart failure receiving inotropic therapy. Open Heart 2018;5:e000923. doi:10.1136/ openhrt-2018-000923

Received 21 August 2018 Revised 16 October 2018 Accepted 10 November 2018
Check for updates

C Author(s) (or their employer(s)) 2018. Re-use permitted under CC BY-NC. No commercial re-use. See rights and permissions. Published by BMJ.

${ }^{1}$ Internal Medicine Department, Centro Hospitalar de São João, Porto, Portugal

${ }^{2}$ Heart Institute (InCor) do Hospital das Clínicas da Faculdade de Medicina da Universidade de São Paulo, São Paulo, Brazil

Correspondence to Dr Victor Sarli Issa; victor.issa@ incor.usp.br

\section{ABSTRACT}

Objectives The prognostic significance of transient use of inotropes has been sufficiently studied in recent heart failure (HF) populations. We hypothesised that risk stratification in these patients could contribute to patient selection for advanced therapies.

Methods We analysed a prospective cohort of adult patients admitted with decompensated HF and ejection fraction (left ventricular ejection fraction (LVEF)) less than $50 \%$. We explored the outcomes of patients requiring inotropic therapy during hospital admission and after discharge.

Results The study included 737 patients, (64.0\% male), with a median age of 58 years (IQR 48-66 years). Main aetiologies were dilated cardiomyopathy in 273 (37.0\%) patients, ischaemic heart disease in 195 (26.5\%) patients and Chagas disease in 163 (22.1\%) patients. Median LVEF was $26 \%$ (IQR 22\%-35\%). Inotropes were used in 518 (70.3\%) patients. In 431 (83.2\%) patients, a single inotrope was administered. Inotropic therapy was associated with higher risk of in-hospital death/urgent heart transplant $(\mathrm{OR}=10.628,95 \% \mathrm{Cl} 5.055$ to $22.344, \mathrm{p}<0.001)$. At 180 day follow-up, of the 431 patients discharged home, 39 $(9.0 \%)$ died, 21 (4.9\%) underwent transplantation and 183 $(42.4 \%)$ were readmitted. Inotropes were not associated with outcome (death, transplant and rehospitalisation) after discharge.

Conclusions Inotropic drugs are still widely used in patients with advanced decompensated HF and are associated with a worse in-hospital prognosis. In contrast with previous results, intermittent use of inotropes during hospitalisation did not determine a worse prognosis at 180-day follow-up. These data may add to prognostic evaluation in patients with advanced $\mathrm{HF}$ in centres where mechanical circulatory support is not broadly available.

\section{INTRODUCTION}

Hospital admissions are frequent among patients with heart failure $(\mathrm{HF})^{1}$ with a broad range of clinical presentations and haemodynamic profiles. It has been estimated that half of patients have either arterial hypotension or other signs of tissue hypoperfusion at hospital admission, ${ }^{23}$ and a significant

\section{Key questions}

What is already known about this subject?

- Inotropes have been consistently associated with worse prognosis when administered in the absence of cardiogenic shock or tissue hypoperfusion.

What does this study add?

- Inotropic used in patients with advanced decompensated HF is associated with worse in-hospital prognosis. However, transient use of inotropes during hospitalisation did not determine a worse prognosis at 180 -day follow-up.

How might this impact on clinical practice?

Inotropic therapy may not be a good marker for selection of candidates of advanced heart failure therapies, such as ventricular assist devices, especially in centres where these therapies are not broadly available.

proportion of these patients receive inotropic therapy during the course of hospitalisation. ${ }^{4}$

Inotropes have been consistently associated with worse prognosis when administered in the absence of cardiogenic shock or tissue hypoperfusion, ${ }^{56}$ and suggested mechanisms involve increased risk for ventricular arrhythmias, increased myocardial metabolic demand, eosinophilic myocarditis and interactions with other medical interventions, such as beta-blocker therapy. ${ }^{7}$ Current indications for administration of inotropes remain restricted to the presence of cardiogenic shock and persistent signs of tissue hypoperfusion. ${ }^{8}$ In this scenario, patients who become inotropic dependent during episodes of decompensated HF have limited therapeutic options and are considered potential candidates for advanced therapies and palliative care; specifically, inotropic dependency is currently a criterion for selection of patients who may benefit from mechanical circulatory support (MCS) systems. ${ }^{8}$ 
However, recent studies evaluating the effect on inotropic therapy in patients with advanced HF and persistent states of low cardiac output have suggested that this therapeutic strategy may be safe and that patient outcomes may have improved in the face of contemporary HF medical treatment, as well as due to the increased number of ICDs in these populations. ${ }^{9}$ Furthermore, the prognostic significance of transient use of inotropes has not been sufficiently studied in recent patient populations. This information may be especially valuable in centres where advanced HF therapies, such as heart transplants or MCSs, are not broadly available, as these therapies are frequently restricted by a limited donor supply, presence of comorbidities or lack of social and economic support.

Therefore, we hypothesised that risk stratification in patients who are eventually weaned from inotropes during episodes of acute decompensated HF could contribute for better patient selection for advanced HF therapies.

\section{METHODS \\ Objectives}

The primary aim of our study was to analyse the outcomes of patients requiring inotropic therapy during the course of a hospital admission due to decompensated HF, as well as 180 days after hospital discharge. Furthermore, we sought to identify clinical variables possibly associated with a less favourable outcome in this patient population.

\section{Study design}

We analysed a prospective cohort of patients admitted to the Heart Institute (InCor) of the Hospital das Clinicas da Faculdade de Medicina da Universidade de São Paulo with a diagnosis of acute decompensated HF. The first inclusion occurred in August 2013, and the last was in December 2017. Patients were followed up for 180 days after hospital discharge.

\section{Patients}

We included patients over 18 years of age admitted with a diagnosis of acute decompensated HF and ejection fraction less than $50 \%$ as measured by echocardiography. We excluded patients hospitalised for less than 24 hours and patients with cardiogenic shock or decompensated HF during the postoperative period after heart surgery. For the purpose of the present analysis, HF aetiology was categorised into three groups as follows: Chagas cardiomyopathy, ischaemic cardiomyopathy and dilated cardiomyopathy (DCM) related to other conditions.

\section{Variables}

Data were obtained from medical records, including demographic information, epidemiological data, pathological history, reason for hospitalisation, presence and duration of HF-related symptoms, aetiological diagnosis of HF or cardiomyopathy, physical examination, electrocardiographic and echocardiographic data and major events during hospitalisation, that is, death and heart transplantation. After hospital discharge, we recorded the occurrence of death, heart transplantation or readmission at 180 days.

\section{Statistical analysis}

Categorical variables are described as absolute value and percentage; continuous variables are described as median \pm IQR 25\%-75\%. For non-normal distribution of variables, the non-parametric Mann-Whitney $\mathrm{U}$ test was used. Comparison of proportions between groups was performed with the $\chi^{2}$ test. Multivariate analysis was performed with stepwise logistic regression. Survival was estimated by using the Kaplan-Meier method, and differences in survival between groups were assessed with the log-rank test. Cox proportional hazards models were used to determine the influence of the variables on patients' survival. We included in the model variables with a $p$ value in univariate analysis less than 0.1 . $P$ values less than 0.05 were considered significant. Statistical analysis was performed using SPSS for Windows 11.0.

\section{RESULTS}

\section{Baseline characteristics}

We included 737 patients admitted with decompensated HF from August 2013 to December 2017 (table 1). Patients were predominantly male $(64.0 \%)$, and the median age was 58 years (IQR 48-66 years). Main aetiologies were DCM in $273(37.0 \%)$ patients, ischaemic heart disease in $195(26.5 \%)$ patients and Chagas disease in 163 (22.1\%) patients. Median left ventricular ejection fraction (LVEF) was 26\% (IQR 22\%-35\%). Inotropes were used in 518 (70.3\%) patients: dobutamine in 494 (95.4\%), milrinone in $88(17.0 \%)$ and levosimendan in $17(3.3 \%)$ patients. In most patients $(431,83.2 \%)$, a single inotropic agent was administered (dobutamine in 97\%), and association of inotropic drugs (dobutamine with milrinone and/ or levosimendan) was observed in $16.8 \%$ (87 patients). Vasopressor support with norepinephrine was used in $169(32.6 \%)$ patients.

\section{Comparison of clinical and laboratory variables}

When clinical characteristics were analysed according to the use inotropic drugs during hospital stay (table 1), we found that patients who required inotropic therapy frequently had a higher proportion of HF secondary to Chagas and valvular disease $(25.7 \%$ vs $13.7 \%$ and $6.0 \%$ vs $2.7 \%$, respectively, $\mathrm{p}<0.001$ ), and a precipitant factor for HF decompensation was less frequently identified $(46.5 \%$ vs $68.0 \%$, respectively, $\mathrm{p}<0.001)$. At admission, patients requiring inotropic therapy more often had clinical and laboratory signs of both congestion and decreased organ perfusion; furthermore, median LVEF was also lower (25\% (IQR 21-30) vs 30\% (25-40), respectively, $\mathrm{p}<0.001$ ) and RV dysfunction was more frequent (44.8\% vs $21.0 \%$, respectively, $\mathrm{p}<0.001)$ in these patients. 
Table 1 Baseline characteristics of patients

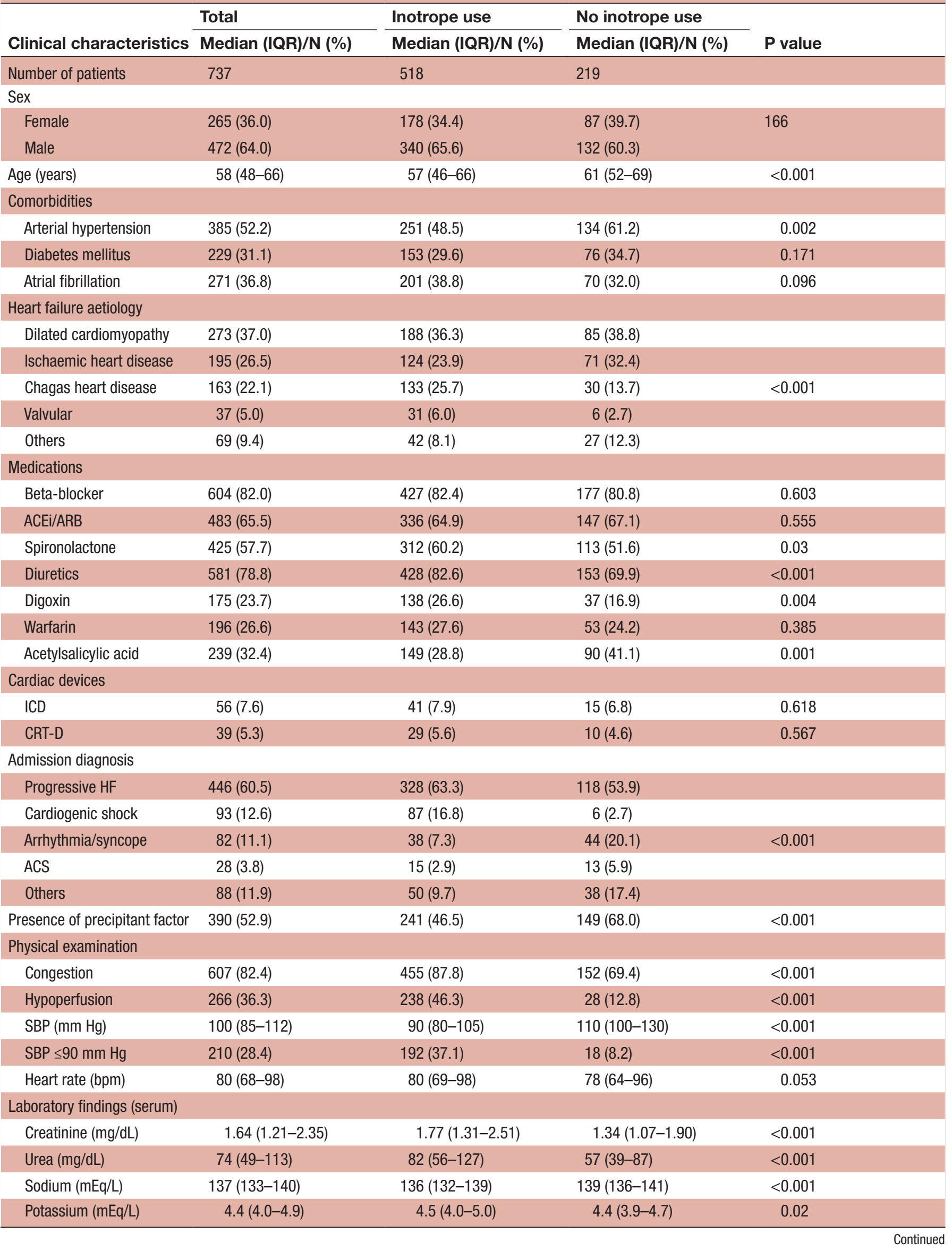




\begin{tabular}{|c|c|c|c|c|}
\hline & Total & Inotrope use & No inotrope use & \\
\hline Clinical characteristics & Median (IQR)/N (\%) & Median (IQR)/N (\%) & Median (IQR)/N (\%) & $P$ value \\
\hline BNP (pg/dL) & 1089 (472-2025) & 1239 (606-2201) & $595(291-1335)$ & $<0.001$ \\
\hline \multicolumn{5}{|l|}{ Echocardiographic findings } \\
\hline LV ejection fraction (\%) & $26(22-35)$ & $25(21-30)$ & $30(25-40)$ & $<0.001$ \\
\hline RV disfunction & $278(37.7)$ & $232(44.8)$ & $46(21.0)$ & $<0.001$ \\
\hline
\end{tabular}

ACEi, ACE inhibitor; ACS, acute coronary syndrome; ARB, angiotensin receptor blocker; BNP, B natriuretic peptide; CRT-D, defibrillator with cardiac resynchronisation therapy; HF, heart failure; ICD, implantable cardioverter defibrillator; LV, left ventricle; RV, right ventricle; SBP, systolic blood pressure.

\section{In-hospital prognosis}

During hospital stay, 215 (29.2\%) patients died, and 87 $(11.8 \%)$ underwent heart transplantation. Patients who required inotropic support had a higher proportion of in-hospital death and heart transplantation, compared with patients who did not require inotropic support ( $40.3 \%$ vs $3.2 \%$ and $15.9 \%$ vs $2.3 \%$, respectively, $\mathrm{p}<0.001$ ) (figure 1); $228(44.0 \%)$ were successfully weaned off inotropes and discharged.

In a logistic regression analysis for adverse in-hospital composite outcome (death plus heart transplantation) (table 2), inotropic therapy was associated with a 10.6fold higher risk of composite outcome ( $\mathrm{OR}=10.628,95 \%$ CI 5.055 to $22.344, \mathrm{p}<0.001)$. Other independent variables significantly associated with composite outcome were $\mathrm{HF}$, with ischaemic disease being associated with a twofold increased risk of adverse outcome compared with DCM (OR=1.961, 95\% CI 1.128 to 3.410, $\mathrm{p}=0.017)$, lower $\mathrm{SBP}$ on admission $(\mathrm{OR}=0.985,95 \%$ CI 0.975 to 0.995 , $\mathrm{p}=0.003)$ and higher $\mathrm{B}$ natriuretic peptide (BNP) level on admission (OR $1.215,95 \%$ CI 1.040 to $1.420, \mathrm{p}=0.014$ ).

We further separately analysed patients who required inotropic support and found that, compared with discharged patients, those who had composite outcome (death or heart transplant) had more signs of congestion
$(91.4 \%$ vs $83.3 \%$, respectively, $\mathrm{p}=0.005)$, lower SBP (median 90 (IQR 80-100) mm Hg vs 98 (IQR 84-110), $\mathrm{p}=0.001$ ), lower heart rate (median 80 (IQR 68-96) bpm vs 84 (IQR 70-103) bpm, $\mathrm{p}=0.040$ ), higher serum urea level (median 88 (IQR 57-139) mg/dL vs 77 (IQR $55-113) \mathrm{mg} / \mathrm{dL}, \mathrm{p}=0.029)$, lower serum sodium level (median 135 (IQR 132-138) mEq/L vs 137 (IQR 133-139) $\mathrm{mEq} / \mathrm{L}, \mathrm{p}=0.004$ ) and higher BNP level (median 1419 (IQR 606-2195) pg/dL vs 1037 (IQR 469-1796) pg/ $\mathrm{dL}, \mathrm{p}<0.001$ ) at admission, lower LVEF (median 25\% (IQR 20.30) vs 28 (IQR 24-32), p<0.001) and higher proportion of RV dysfunction ( $51.4 \%$ vs $36.4 \%, \mathrm{p}=0.001$ ) (table 3). The proportion of death and heart transplantation was especially high among patients treated with an association of different inotropic drugs during hospital stay (figure 2).

In multivariable analysis among patients who underwent inotropic support, variables associated with composite outcome were ischaemic aetiology (compared with DCM, OR 1.992, 95\% CI 1.091 to 3.635, $\mathrm{p}=0.025$ ), lower admission SBP (OR=0.986, 95\% CI 0.975 to 0.996$)$, higher admission BNP level (OR=1.193, 95\% CI 1.009 to 1.411, $\mathrm{p}=0.039$ ) and presence of association of different inotropes $(\mathrm{OR}=5.524,95 \%$ CI 2.692 to $11.335, \mathrm{p}<0.001)$ (table 2).

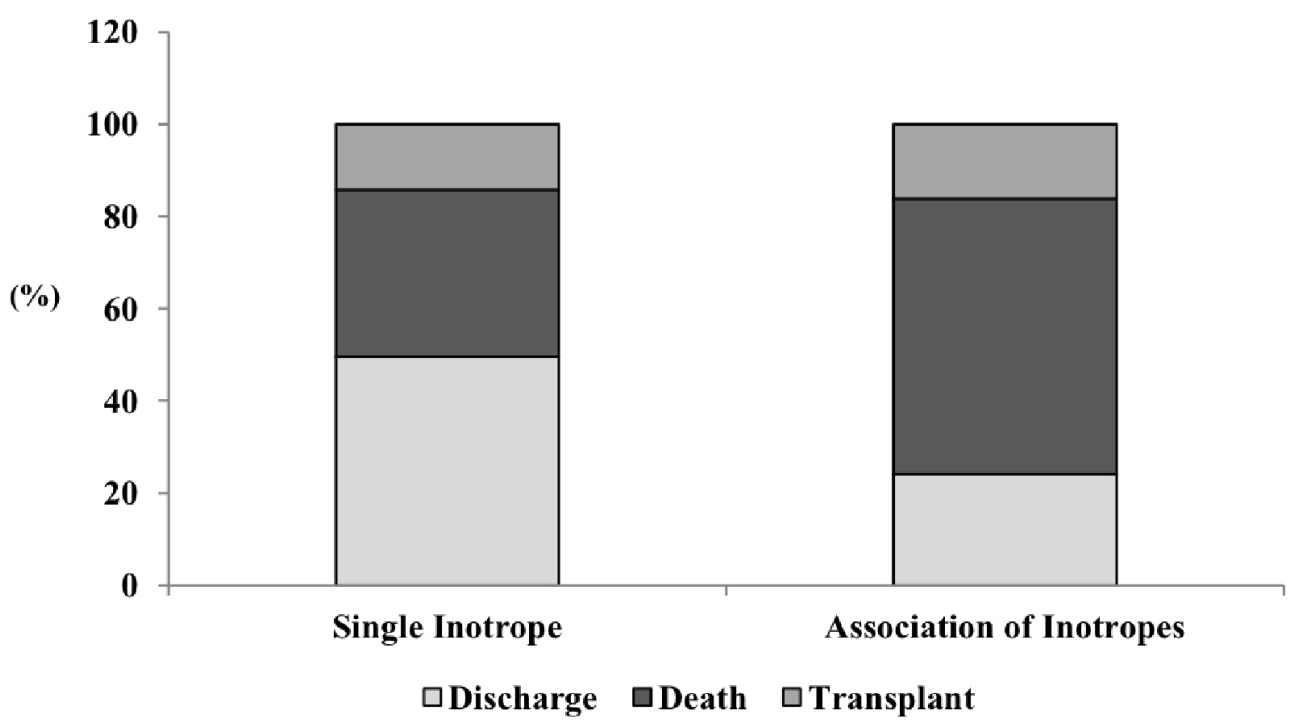

Figure 1 In-hospital outcomes (discharge, death, or heart transplantation) according to treatment with inotropic drug. 
Table 2 Multivariable analysis of clinical and laboratory findings for the occurrence of death or heart transplantation during hospital stay

\begin{tabular}{|c|c|c|c|}
\hline & OR & $95 \% \mathrm{Cl}$ & $P$ value \\
\hline \multicolumn{4}{|l|}{ All patients } \\
\hline Age & 0.995 & 0.979 to 1.011 & 0.551 \\
\hline Arterial hypertension & 0.747 & 0.473 to 1.180 & 0.211 \\
\hline Diabetes mellitus & 1.412 & 0.874 to 2.280 & 0.159 \\
\hline Ischaemic aetiology & 1.961 & 1.128 to 3.410 & 0.017 \\
\hline Decompensation factor & 0.855 & 0.573 to 1.277 & 0.444 \\
\hline LV ejection fraction & 0.978 & 0.955 to 1.002 & 0.072 \\
\hline RV dysfunction & 1.359 & 0.890 to 2.076 & 0.155 \\
\hline \multicolumn{4}{|l|}{ Admission data: } \\
\hline SBP & 0.985 & 0.975 to 0.995 & 0.003 \\
\hline Heart rate & 0.996 & 0.987 to 1.005 & 0.364 \\
\hline BNP & 1.215 & 1.040 to 1.420 & 0.014 \\
\hline Blood urea & 1.003 & 0.999 to 1.007 & 0.109 \\
\hline Inotropic drug use & 10.628 & 5.055 to 22.344 & $<0.001$ \\
\hline \multicolumn{4}{|c|}{ Patients treated with inotropics } \\
\hline Age & 1 & 0.982 to 1.017 & 0.967 \\
\hline Arterial hypertension & 0.701 & 0.423 to 1.161 & 0.168 \\
\hline Diabetes mellitus & 1.378 & 0.813 to 2.334 & 0.234 \\
\hline Ischaemic aetiology & 1.992 & 1.091 to 3.635 & 0.025 \\
\hline Decompensation factor & 0.95 & 0.614 to 1.471 & 0.819 \\
\hline LV ejection fraction & 0.975 & 0.950 to 1.002 & 0.067 \\
\hline RV dysfunction & 1.526 & 0.964 to 2.417 & 0.071 \\
\hline \multicolumn{4}{|l|}{ Admission data: } \\
\hline SBP & 0.986 & 0.975 to 0.996 & 0.008 \\
\hline Heart rate & 0.99 & 0.981 to 1.000 & 0.044 \\
\hline BNP & 1.193 & 1.009 to 1.411 & 0.039 \\
\hline Blood urea & 1.002 & 0.998 to 1.005 & 0.436 \\
\hline Association of inotropes & 5.524 & 2.692 to 11.335 & $<0.001$ \\
\hline
\end{tabular}

BNP, B natriuretic peptide; LV, left ventricle; RV, right ventricle; SBP, systolic blood pressure.

\section{Prognosis after hospital discharge}

During 180 days of follow-up, of the 431 patients discharged home, 39 (9.0\%) died, 21 (4.9\%) underwent transplantation and $183(42.4 \%)$ were readmitted. The use of inotropes was not associated with composite outcome (death, transplant and rehospitalisation) in an unadjusted analysis (HR 0.965 ; 95\% CI 0.687 to 1.355 ; $\mathrm{p}=0.836$ ) (figure 3). From the patients who had been treated with inotropes during hospitalisation, $82.6 \%$ were alive at 180 days with no heart transplant or need for MCSs. In a model of multivariate regression adjusted for age, of the variables HF aetiology, presence of precipitant factor, arterial hypertension, diabetes mellitus, LVEF, right ventricular dysfunction at echocardiography, use of inotropes during hospitalisation, as well as BNP and urea at discharge, the only variable independently associated with composite outcome was LVEF (HR $=0.847,95 \%$ CI 0.743 to $0.966, \mathrm{p}=0.013)$.

\section{DISCUSSION}

In the present study, we explored the impact of inotropic therapy in patients with decompensated HF and the importance of further risk stratification in this patient population. We found that inotropic therapy is a strong predictor of in-hospital death and that clinical variables, such as ischaemic aetiology, admission blood pressure, BNP value and use of more than one inotropic agent, can offer additional risk to these patients. However, in our cohort, inotropic therapy during hospital admission was not associated with a worse prognosis after discharge. Importantly, we found in our population a high rate of inotropic therapy and a high rate of in-hospital death and heart transplantation.

The proportion of patients using inotropes during hospitalisation for decompensated $\mathrm{HF}$ is variable among studies, about $7 \%$ to $42 \%^{46^{10-13}}$ and the indication for their use is not always clear. In a prospective, multicentre, observational study ${ }^{4}$ of 1855 patients admitted with acute $\mathrm{HF}$, inotropes were used in $19.4 \%$ of patients, and about $50 \%$ of patients receiving inotropes had systolic blood pressure at admission $>100 \mathrm{~mm} \mathrm{Hg}$ and only $27 \%$ of these showed signs of hypoperfusion. In the Organized Program to Initiate Lifesaving Treatment in Hospitalized Patients with Heart Failure (OPTIMIZE-HF) registry, ${ }^{14}$ which included 48612 patients hospitalised with acute HF, inotropes were used in $7 \%$ and about $48 \%$ had a systolic blood pressure $\geq 120 \mathrm{~mm} \mathrm{Hg}$ on admission. In the The Acute Decompensated Heart Failure National Registry (ADHERE) registry, ${ }^{6}$ only about $8 \%$ of patients treated with inotropes had systolic blood pressure $<90 \mathrm{~mm} \mathrm{Hg}$ at admission. These results may suggest that the use of inotropes may not always be performed according to the current guidelines, which state that intravenous inotropic drugs are appropriate for short-term use in patients with SBP $<90 \mathrm{~mm} \mathrm{Hg}$ and/or signs/symptoms of hypoperfusion (class IIb recommendation). ${ }^{8}$ In our study, $64 \%$ of patients showed signs of hypoperfusion and/or SBP $<90$ $\mathrm{mm} \mathrm{Hg}$, and positive intravenous inotropic drugs were used in $70 \%$ of the total population. It should be noted that systolic blood pressure and hypoperfusion data were collected on admission, while inotropic drugs could have been introduced later during the course of hospitalisation with the patient presenting with a different haemodynamic profile. The higher percentage of inotropic use in our hospital may be explained by the severity of the disease in this population, as shown by the low median LVEF $(26 \%)$, high proportion of right ventricle dysfunction $(37.7 \%)$, high median creatinine level $(1.64 \mathrm{mg} /$ $\mathrm{dL}$ ) and the need for cardiac transplantation during hospitalisation and follow-up in $14.8 \%$ of patients; additionally, the study was performed in a tertiary hospital to which refractory patients are referred for advanced HF therapies. 
Table 3 Baseline characteristics in the group of patients under inotropic therapy

\begin{tabular}{|c|c|c|c|c|}
\hline & Total & Death/HTx & Discharge & \\
\hline Clinical characteristics & Median (IQR)/N (\%) & Median (IQR)/N (\%) & $\begin{array}{l}\text { Median (IQR)/N } \\
(\%)\end{array}$ & $P$ value \\
\hline Number of patients & 518 & 290 & 228 & \\
\hline \multicolumn{5}{|l|}{ Sex } \\
\hline Female & $178(34.4)$ & $106(36.6)$ & $72(31.6)$ & 0.237 \\
\hline Male & $340(65.6)$ & $184(63.4)$ & $156(68.4)$ & \\
\hline Age (years) & $57(46-66)$ & $56(45-65)$ & $58(47-67)$ & 0.269 \\
\hline \multicolumn{5}{|l|}{ Comorbidities } \\
\hline Arterial hypertension & $251(48.5)$ & $133(45.9)$ & $118(51.8)$ & 0.218 \\
\hline Diabetes mellitus & $153(29.6)$ & $91(31.5)$ & $62(27.2)$ & 0.288 \\
\hline Atrial fibrillation & $201(39.3)$ & $119(41.6)$ & $82(36.3)$ & 0.22 \\
\hline \multicolumn{5}{|l|}{ Heart failure aetiology } \\
\hline Dilated cardiomyopathy & $188(36.3)$ & $98(33.8)$ & $90(39.5)$ & \\
\hline Ischaemic heart disease & $124(23.9)$ & $75(25.9)$ & $49(21.5)$ & \\
\hline Chagas heart disease & $132(25.7)$ & $81(27.9)$ & $52(22.8)$ & 0.245 \\
\hline Valvular & $31(6.0)$ & $17(5.9)$ & $14(6.1)$ & \\
\hline Others & $42(8.1)$ & $19(6.6)$ & $23(10.1)$ & \\
\hline \multicolumn{5}{|l|}{ Medications } \\
\hline Beta-blocker & $427(82.4)$ & $241(83.1)$ & $186(81.6)$ & 0.651 \\
\hline ACEi/ ARB & $336(64.9)$ & $193(66.6)$ & $143(62.7)$ & 0.364 \\
\hline Spironolactone & $312(60.2)$ & $180(62.1)$ & $132(57.9)$ & 0.335 \\
\hline Diuretics & $428(82.6)$ & $248(85.5)$ & $180(78.9)$ & 0.05 \\
\hline Digoxin & $138(26.6)$ & $88(30.3)$ & $50(21.9)$ & 0.032 \\
\hline Warfarin & $143(27.6)$ & $86(29.7)$ & $57(25.0)$ & 0.239 \\
\hline Acetylsalicylic acid & $149(28.8)$ & $80(27.6)$ & $69(30.3)$ & 0.504 \\
\hline \multicolumn{5}{|l|}{ Cardiac devices } \\
\hline ICD & $41(7.9)$ & $30(10.3)$ & $11(4.8)$ & 0.021 \\
\hline CRT-D & $29(5.6)$ & $18(6.2)$ & $11(4.8)$ & 0.497 \\
\hline \multicolumn{5}{|l|}{ Admission diagnosis } \\
\hline Progressive HF & $328(63.3)$ & $176(60.7)$ & $155(66.7)$ & \\
\hline Cardiogenic shock & 87 (16.9) & $56(19.3)$ & $31(13.7)$ & \\
\hline Arrhythmia/syncope & $38(7.4)$ & $22(7.6)$ & $16(7.1)$ & 0.213 \\
\hline ACS & $15(2.9)$ & $11(3.8)$ & $4(1.8)$ & \\
\hline Others & $50(9.7)$ & $25(8.6)$ & $25(11.0)$ & \\
\hline Presence of precipitant factor & $241(46.7)$ & $128(44.1)$ & $113(50.0)$ & 0.185 \\
\hline \multicolumn{5}{|l|}{ Physical examination } \\
\hline Congestion & $455(87.8)$ & $265(91.4)$ & $190(83.3)$ & 0.005 \\
\hline Hypoperfusion & $238(46.3)$ & $139(48.1)$ & $99(44.0)$ & 0.355 \\
\hline $\mathrm{SBP}(\mathrm{mm} \mathrm{Hg})$ & $90(80-105$ & $90(80-100)$ & $98(84-110)$ & 0.001 \\
\hline Heart rate (bpm) & $80(69-98)$ & $80(68-96)$ & $84(70-103)$ & 0.04 \\
\hline \multicolumn{5}{|l|}{ Laboratory findings (serum) } \\
\hline Creatinine (mg/dL) & $1.76(1.31-2.52)$ & $1.81(1.39-2.64)$ & $1.69(1.27-2.49)$ & 0.13 \\
\hline Urea (mg/dL) & $82(56-127)$ & $88(57-139)$ & $77(55-113)$ & 0.029 \\
\hline Sodium (mEq/L) & 136 (132-139) & 135 (132-138) & 137 (133-139) & 0.004 \\
\hline Potassium (mEq/L) & $4.5(4.0-5.0)$ & $4.5(4.0-5.0)$ & $4.5(3.9-5.0)$ & 0.717 \\
\hline
\end{tabular}


Table 3 Continued

\begin{tabular}{|c|c|c|c|c|}
\hline \multirow[b]{2}{*}{ Clinical characteristics } & Total & Death/HTx & Discharge & \multirow[b]{2}{*}{$P$ value } \\
\hline & Median (IQR)/N (\%) & Median (IQR)/N (\%) & $\begin{array}{l}\text { Median (IQR)/N } \\
(\%)\end{array}$ & \\
\hline BNP (pg/dL) & $1236(606-2195)$ & $1419(747-2372)$ & $1037(469-1796)$ & $<0.001$ \\
\hline \multicolumn{5}{|l|}{ Echocardiographic findings } \\
\hline LV ejection fraction (\%) & $25(21-30)$ & $25(20-30)$ & $28(24-32)$ & $<0.001$ \\
\hline RV dysfunction & $232(44.8)$ & $149(51.4)$ & $83(36.4)$ & 0.001 \\
\hline
\end{tabular}

ACEi, ACE inhibitor; ACS, acute coronary syndrome; ARB, angiotensin receptor blocker; BNP, B natriuretic peptide; CRT-D, defibrillator with cardiac resynchronisation therapy; HF, heart failure; HTx, heart transplant; ICD, implantable cardioverter defibrillator; LV, left ventricle; RV, right ventricle; SBP, systolic blood pressure.

Similar to previous results, in-hospital mortality was significantly higher in the patients treated with inotropes (40.2\% vs $3.2 \%$ ), with inotropic stimulation being the strongest predictor of outcome during hospitalisation (10-fold increased risk of death/heart transplantation). Other variables associated with outcome were ischaemic HF aetiology, lower systolic blood pressure and higher BNP level on admission (table 2). Among patients treated with inotropes, association of different positive inotropic drugs had a fivefold increased risk of composite outcome. A recent retrospective cohort of 500 adult patients treated with dobutamine or milrinone during hospitalisation for acute decompensated $\mathrm{HF}^{14}$ had an overall 180-day mortality rate of $16 \%$. Seemingly, post hoc analysis of Acute Heart Failure Global Survey of Standard Treatment (ALARM-HF) data ${ }^{15}$ showed a much greater in-hospital mortality rate in patients receiving intravenous inotropes $(25.9 \%)$ compared with those who did not $(5.2 \%)$, regardless of the admission SBP. After propensity-based matching, they found a 1.5-fold increased risk of death in patients receiving dopamine or dobutamine compared with patients not receiving inotropes. Furthermore, an analysis of the ADHERE registry ${ }^{6}$ found an increased in-hospital mortality associated with treatment with dobutamine or milrinone compared with nitroglycerin or nesiritide $(12.3 \%$ and $13.9 \%$ vs $4.7 \%$ and $7.1 \%$, respectively). In the OPTIMIZE-HF registry, in-hospital mortality was associated with lower SBP on admission and independently with the use of inotropes. In fact, even when systolic blood pressure was $>120 \mathrm{~mm} \mathrm{Hg}$, mortality was higher in the patients treated with these drugs. Besides lower systolic blood pressure and inotropic stimulation, other variables independently associated with in-hospital mortality in other studies were older age, serum creatinine $>1.5 \mathrm{mg} / \mathrm{dL}$ and serum sodium $<136 \mathrm{mEq} / \mathrm{L}$.

In our study, a significant proportion of patients $(44 \%)$ were weaned off inotropes and discharged home. Of these, 190 patients $(82.6 \%)$ were alive at 180 days with no heart transplant or need for MCSs. Other authors reported similar results in a population of 80 patients dependent on inotropic support, where about $55 \%$ of patients were weaned-off inotropes, most of them being discharged home and showing an LVAD/transplant-free cumulative survival of $71 \%$, during a mean follow-up of 2074 days. $^{16}$ In our population, no difference existed in all-cause mortality, need for heart transplantation

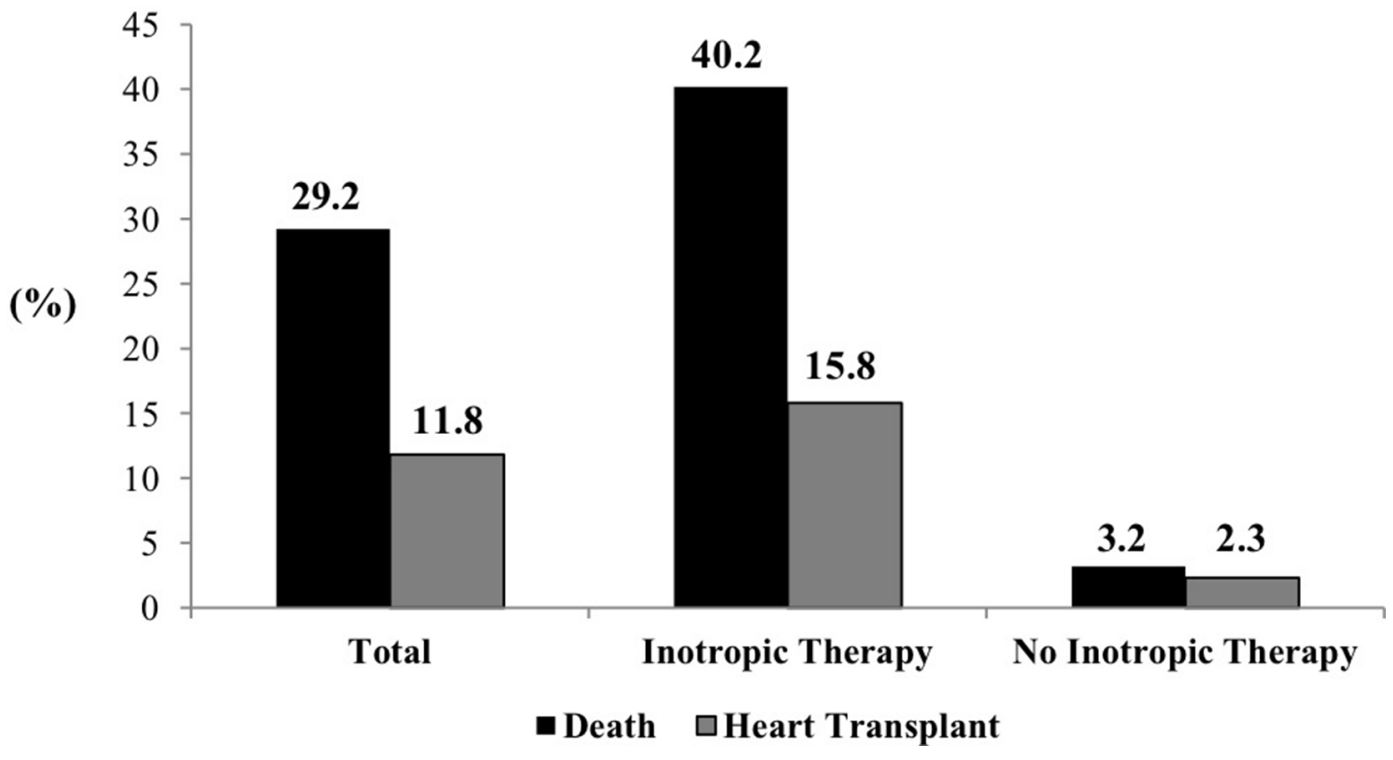

Figure 2 In-hospital outcome according to the number inotropic drugs used. 


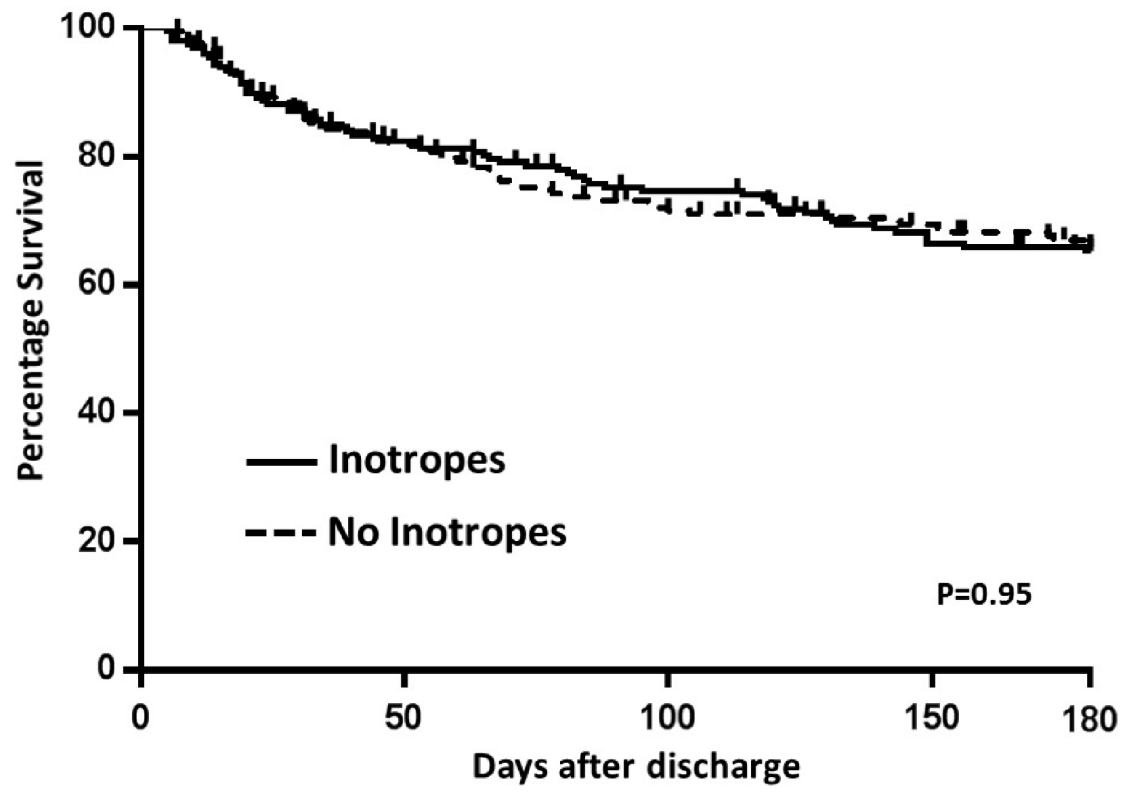

$\begin{array}{lr}\text { Number of patients at risk } \\ \text { Inotropes } & 199 \\ \text { No inotropes } & 216\end{array}$

154

165
133

136
116

124
107

115

Figure 3 Kaplan-Meier survival curve for composite outcome (hospitalisation, heart transplantation or death) at 180 days of follow-up, according to treatment with inotropic drugs.

or rehospitalisation after discharge. Patients who were weaned off inotropes showed a similar outcome, compared with those who did not need inotropic support, and the only variable we found to be related to composite outcome was a lower LVEF. Studies on out-of-hospital prognosis after treatment with inotropic drugs in the acute setting are rare, and most of them support the harmful impact of these drugs. Another study found that both in-hospital mortality and mortality at 1-year follow-up were higher in the patients treated with inotropes during hospitalisation $(21.4 \%$ vs $2.7 \%$ and $50.6 \%$ vs $17.7 \%$, respectively), and inotropic use was independently associated with all-cause mortality using a propensity score adjustment logistic regression. ${ }^{4}$ A post hoc analysis of the Evaluation Study of Congestive Heart Failure and Pulmonary Artery Catheterization Effectiveness (ESCAPE) trial also showed a shorter survival after discharge among patients who received in-hospital inotropes, compared with those who did not (144 vs 165 median days, respectively). ${ }^{17}$

Finally, it should be noted that most of the evidence about the impact of inotropes on HF comes from observational studies or post hoc analysis of trials not designed for the purpose. In fact, the severity of the disease and the dependence on inotropes for survival represent clinical and ethical restrictions to the development of randomised placebo controlled trials in this setting. Furthermore, most of these trials were performed before ICDs were used for primary prevention (which could now protect from inotropic-associated mortality) and used inotropic drugs that are no longer in use. Therefore, we have insufficient evidence to support conclusions about inotrope safety nowadays.

Limitations of this study are mostly related to its design: because data were obtained from medical records, it sometimes resulted in incomplete clinical and haemodynamic data, and information about cause of death was not always available. Timing and dose of the inotrope used were not available and could have allowed a better analysis of the association of these drugs with the outcome. The single-centre nature of our study may also reflect local practice patterns, and comparison with other populations should be done with caution.

In conclusion, inotropic drugs are still widely used for support of patients with advanced decompensated HF and are associated with worse in-hospital prognosis. However, transient use of inotropes during hospitalisation did not determine a worse prognosis at 180-day follow-up, and thus, was not a good marker for selection of candidates of advanced HF therapies, such as MCSs, especially in centres where these therapies are not broadly available.

Contributors CG contributed to conception and design of the research, acquisition of data, analysis and interpretation of data, statistical analysis, drafting the manuscript and critical revision of the manuscript for important intellectual content. CBT contributed to acquisition of data, statistical analysis and critical revision of the manuscript for important intellectual content. SMA-F contributed to conception and design of the research and critical revision of the manuscript for important intellectual content. GEC-S, VMCS, PRC, MTOJ, SHGL and EAB contributed to conception and design of the research and critical revision of the manuscript for important intellectual content. FF contributed to analysis and interpretation of data, statistical analysis drafting the manuscritp and critical revision of the manuscript for important intellectual content. VSI contributed to conception and design of the 
research, acquisition of data and critical revision of the manuscript for important intellectual content, analysis and interpretation of data, statistical analysis and drafting the manuscript.

Funding The authors have not declared a specific grant for this research from any funding agency in the public, commercial or not-for-profit sectors.

Competing interests None declared.

Patient consent for publication Not required.

Ethics approval The study was approved by the Institutional Ethics Committee for Research Project Analysis of Heart Institute (InCor) of the Hospital das Clínicas da Faculdade de Medicina da Universidade de São Paulo.

Provenance and peer review Not commissioned; externally peer reviewed.

Data sharing statement No additional data are available.

Open access This is an open access article distributed in accordance with the Creative Commons Attribution Non Commercial (CC BY-NC 4.0) license, which permits others to distribute, remix, adapt, build upon this work non-commercially, and license their derivative works on different terms, provided the original work is properly cited, appropriate credit is given, any changes made indicated, and the use is non-commercial. See: http://creativecommons.org/licenses/by-nc/4.0/.

\section{REFERENCES}

1. Arora S, Lahewala S, Hassan Virk HU, et al. Etiologies, trends, and predictors of 30-day readmissions in patients with diastolic heart failure. Am J Cardiol 2017;120:616-24

2. Nohria A, Tsang SW, Fang JC, et al. Clinical assessment identifies hemodynamic profiles that predict outcomes in patients admitted with heart failure. J Am Coll Cardiol 2003;41:1797-804.

3. Terhoch CB, Moreira HF, Ayub-Ferreira SM, et al. Clinical findings and prognosis of patients hospitalized for acute decompensated heart failure: Analysis of the influence of Chagas etiology and ventricular function. PLoS Negl Trop Dis 2018;12:e0006207.

4. Mortara A, Oliva F, Metra M, et al. Treatment with inotropes and related prognosis in acute heart failure: contemporary data from the Italian Network on Heart Failure (IN-HF) Outcome registry. J Heart Lung Transplant 2014;33:1056-65.

5. Cuffe MS, Califf RM, Adams KF, et al. Short-term intravenous milrinone for acute exacerbation of chronic heart failure: a randomized controlled trial. JAMA 2002;287:1541-7.

6. Abraham WT, Adams KF, Fonarow GC, et al. In-hospital mortality in patients with acute decompensated heart failure requiring intravenous vasoactive medications: an analysis from the Acute Decompensated Heart Failure National Registry (ADHERE). J Am Coll Cardiol 2005;46:57-64.

7. von Scheidt W, Pauschinger M, Ertl G. Long-term intravenous inotropes in low-output terminal heart failure? Clin Res Cardiol 2016;105:471-81.

8. Ponikowski P, Voors AA, Anker SD, et al. ESC Guidelines for the diagnosis and treatment of acute and chronic heart failure: The Task Force for the diagnosis and treatment of acute and chronic heart failure of the European Society of Cardiology (ESC). Developed with the special contribution of the Heart Failure Association (HFA) of the ESC. Eur J Heart Fail 2016;2016:891-975.

9. Hashim T, Sanam K, Revilla-Martinez M, et al. Clinical characteristics and outcomes of intravenous inotropic therapy in advanced heart failure. Circ Heart Fail 2015;8:880-6.

10. Follath F, Yilmaz MB, Delgado JF, et al. Clinical presentation, management and outcomes in the Acute Heart Failure Global Survey of Standard Treatment (ALARM-HF). Intensive Care Med 2011;37:619-26.

11. Nieminen MS, Brutsaert D, Dickstein $\mathrm{K}$, et al. EuroHeart Failure Survey II (EHFS II): a survey on hospitalized acute heart failure patients: description of population. Eur Heart $J$ 2006;27:2725-36

12. Gheorghiade M, Abraham WT, Albert NM, et al. Systolic blood pressure at admission, clinical characteristics, and outcomes in patients hospitalized with acute heart failure. JAMA 2006;296:2217-26.

13. Elkayam U, Tasissa G, Binanay C, et al. Use and impact of inotropes and vasodilator therapy in hospitalized patients with severe heart failure. Am Heart J 2007:153:98-104.

14. King JB, Shah RU, Sainski-Nguyen A, et al. Effect of Inpatient Dobutamine versus Milrinone on Out-of-Hospital Mortality in Patients with Acute Decompensated Heart Failure. Pharmacotherapy 2017;37:662-72.

15. Mebazaa A, Parissis J, Porcher R, et al. Short-term survival by treatment among patients hospitalized with acute heart failure: the global ALARM-HF registry using propensity scoring methods. Intensive Care Med 2011;37:290-301.

16. Constantinescu AA, Caliskan K, Manintveld OC, et al. Weaning from inotropic support and concomitant beta-blocker therapy in severely ill heart failure patients: take the time in order to improve prognosis. Eur J Heart Fail 2014;16:435-43.

17. Kalogeropoulos AP, Marti CN, Georgiopoulou VV, et al. Inotrope use and outcomes among patients hospitalized for heart failure: impact of systolic blood pressure, cardiac index, and etiology. J Card Fail 2014;20:593-601. 